\title{
ORIENTATION OF TRANSITION DIPOLE MOMENTS OF RHODAMINE 6G DETERMINED BY EXCITED STATE ABSORPTION
}

\author{
A. PENZKOFER and J. WIEDMANN \\ Fakultät für Physik der Universität Regensburg, D-8400 Regensburg, Germany
}

Received 2 June 1980

Revised manuscript received 15 July 1980

\begin{abstract}
The orientation of the transition dipole moment for $\mathrm{S}_{1}-\mathrm{S}_{4}$ excitation in rhodamine $6 \mathrm{G}$ is measured by excited state absorption with a picosecond pump and probe technique. An orientation parallel to the longitudinal direction of the molecule is found. The result is discussed in terms of molecular symmetry.
\end{abstract}

\section{Introduction}

Fluorescence polarization spectroscopy $\left(\mathrm{S}_{1} \rightarrow \mathrm{S}_{0}\right.$ $\left.[1-4] ; S_{i>1} \rightarrow S_{0}[5-8]\right)$ and absorption dichroism studies $[1,4,9-11]$ are applied to measure the orientation of transition dipole moments. $\mathrm{S}_{0}-\mathrm{S}_{n}$ transition moments of dye molecules are analysed by single photon excitation $[1-4,9,10]$ while $\mathrm{S}_{1}-\mathrm{S}_{n}$ excited state transition moments are determined after a two-step absorption process $[5-8,11]$.

In this letter the orientation of the $\mathrm{S}_{1}-\mathrm{S}_{4}$ transition dipole moment $\boldsymbol{\mu}_{\mathrm{e}}$ of rhodamine $6 \mathrm{G}$ dissolved in ethanol is measured with respect to the orientation of the $\mathrm{S}_{0}-\mathrm{S}_{1}$ transition dipole moment $\boldsymbol{\mu}_{\mathrm{g}}$, i.e. the angle $\alpha$ between $\boldsymbol{\mu}_{\mathrm{e}}$ and $\boldsymbol{\mu}_{\mathrm{g}}$ is determined. A two-step absorption dichroitic study is applied which is similar to the method of [11]. The investigations are carried out at room temperature. The singlet state $\mathrm{S}_{4}$ exhibits ground state absorption around $350 \mathrm{~nm}$ [12]. It is frequently termed as $\mathrm{S}_{2}$ state when two lower lying weakly absorbing singlet levels $[12,25]$ are neglected.

\section{Description}

The angle $\alpha$ between the $S_{1}-S_{4}$ and the $S_{0}-S_{1}$ transition dipole moments is measured with a picosecond pump and probe technique. An intense linearly polarized picosecond pump pulse (duration $\Delta t_{\mathrm{P}} \approx 5 \mathrm{ps,}$ frequency $\tilde{\nu}_{\mathrm{P}}=18960 \mathrm{~cm}^{-1}$, peak intensity $I_{0 \mathrm{P}} \approx$ $10^{9} \mathrm{~W} / \mathrm{cm}^{2}$ ) generates an anisotropic distribution of molecules in the $S_{1}$ state. A probe beam (duration $\Delta t_{\mathrm{L}} \approx 6 \mathrm{ps}$, frequency $\widetilde{v}_{\mathrm{L}}=9480 \mathrm{~cm}^{-1}$ ) passes through the sample immediately after the pump pulse (delay time $t_{\mathrm{D}} \approx 10 \mathrm{ps}$ ) and measures the $\mathrm{S}_{1}-\mathrm{S}_{4}$ excited state absorption. Two sets of transmission measurements are made with the probe beam polarization parallel and perpendicular to the pump pulse polarization. The ratio of the transmissions of the parallel and perpendicular polarized probe pulses at a fixed pump pulse energy determines the angle $\alpha$.

The applied picosecond absorption spectroscopic technique has several advantages:

i) It avoids the problems of orientational brownian motion. In our experiments the orientation time of rhodamine 6G dissolved in ethanol is $\tau_{\text {or }}=270$ ps [13], while the delay time is $t_{\mathrm{D}}=10 \mathrm{ps}$.

ii) High dye concentrations may be used without influence on concentrational depolarization, since the depolarization effects are reduced by a factor of $t_{\mathrm{D}} / \tau_{\mathrm{F}}$ ( $\tau_{\mathrm{F}}$ fluorescence decay time). For rhodamine $6 \mathrm{G}$ it is $\tau_{\mathrm{F}} \approx 4.2$ ns (solvent ethanol) [14] and the critical concentration for conventional fluorescence polarization spectroscopy is $c_{0} \approx 3 \times 10^{-3} \mathrm{M}$ [15].

iii) The problems of triplet state absorption are circumvented because the population of triplet states is negligible within the measurement duration $(\approx 10 \mathrm{ps})$. In case of rhodamine $6 \mathrm{G}$, the $\mathrm{S}_{1}-\mathrm{T}$ intersystem cross- 
(a)

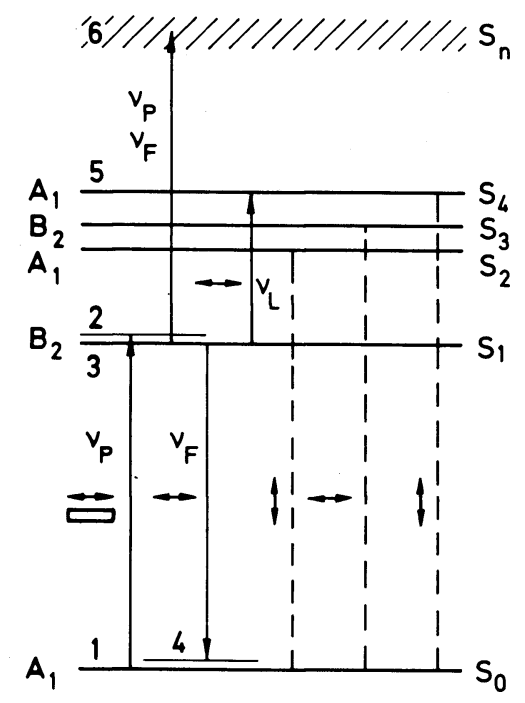

FLUORESCENCE POLARIZÁTION $P$

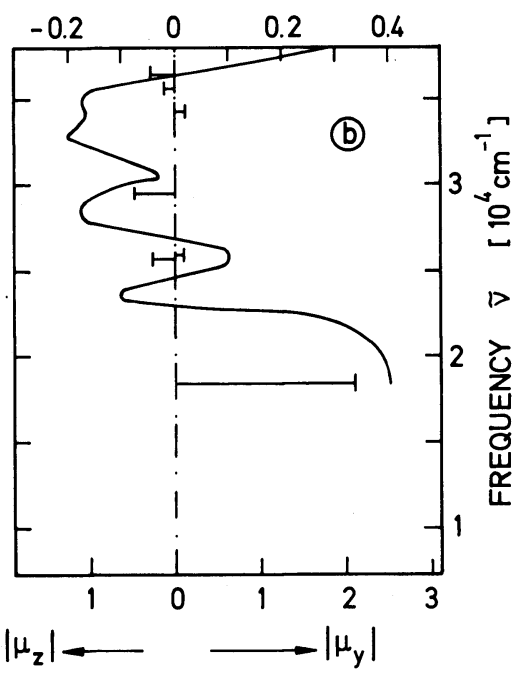

TRANSITION MOMENT [ Debye ]

Fig. 1. (a) Schematic level system of rhodamine 6G. Double arrows $(\leftrightarrow)$ indicate orientations of transition dipole moments relative to molecule ( $\square$ ). (b) Fluorescence polarization spectrum of rhodamine B (after Jacobi and Kuhn [22]) and calculated transition dipole moments parallel $\left(\left|\mu_{y}\right|\right)$ and perpendicular $\left(\left|\mu_{z}\right|\right)$ to longitudinal axis of rhodamine molecules.

rate $k_{\mathrm{ST}} \approx 4.2 \times 10^{5} \mathrm{~s}^{-1}[16]$ is a factor of 500 slower than the $S_{1}-S_{0}$ relaxation rate.

Fig. 1a shows a schematic term system of rhodamine 6G. A pump pulse of frequency $\nu_{\mathrm{P}}$ excites molecules to level 2 where they relax within about 1 ps to the temporal equilibrium position 3 in the $S_{1}$-state [17]. The excited state absorption of the pump pulse and of the generated fluorescence light leads to transitions to region 6. This region is depopulated very fast $\left(\sim 10^{-13} \mathrm{~s}\right)$ and the molecules return preferentially to level 3 [14]. The probe pulses of frequency $\nu_{\mathrm{L}}$ excite molecules from level 3 to 5 .

Fig. 2 shows the orientational arrangement for a pump and probe beam propagating along the same direction ( $y$-axis) and enclosing an angle of $\psi$ between their polarizations $\left(\boldsymbol{E}_{\mathrm{P}}\right.$ and $\left.\boldsymbol{E}_{\mathrm{L}}\right) \cdot \boldsymbol{\mu}_{\mathrm{g}}$ and $\boldsymbol{\mu}_{\mathrm{e}}$ indicate the transition dipole moments of a molecule for pump pulse $\left(S_{0}-S_{1}\right)$ and probe pulses $\left(S_{1}-S_{4}\right)$ absorption, respectively. The moment $\boldsymbol{\mu}_{\mathrm{e}}$ is equally distributed on the conical surface with aperture angle $\alpha$ and axis $\boldsymbol{\mu}_{\mathrm{g}}$ (isotropic probability distribution of $\boldsymbol{\mu}_{\mathrm{e}}$ with respect to angle $\beta$ of fig. 2).

The excited state absorption of the probe pulse depends on the angle $\theta_{\mathrm{e}}$ between $\boldsymbol{\mu}_{\mathrm{e}}$ and $\boldsymbol{E}_{\mathrm{L}}$. The absorption cross-section for electric dipole interaction is $[1,11]$ $\sigma_{\mathrm{e}}\left(\theta_{\mathrm{e}}\right)=3 \sigma_{\mathrm{e}} \cos ^{2}\left(\theta_{\mathrm{e}}\right)$,

$\sigma_{\mathrm{e}}$ is the isotropic excited state absorption cross-section. The angle $\theta_{\mathrm{e}}$ is a function of the angles $\theta_{\mathrm{g}}, \alpha$, and $\beta$. One finds by spherical trigonometry [18]

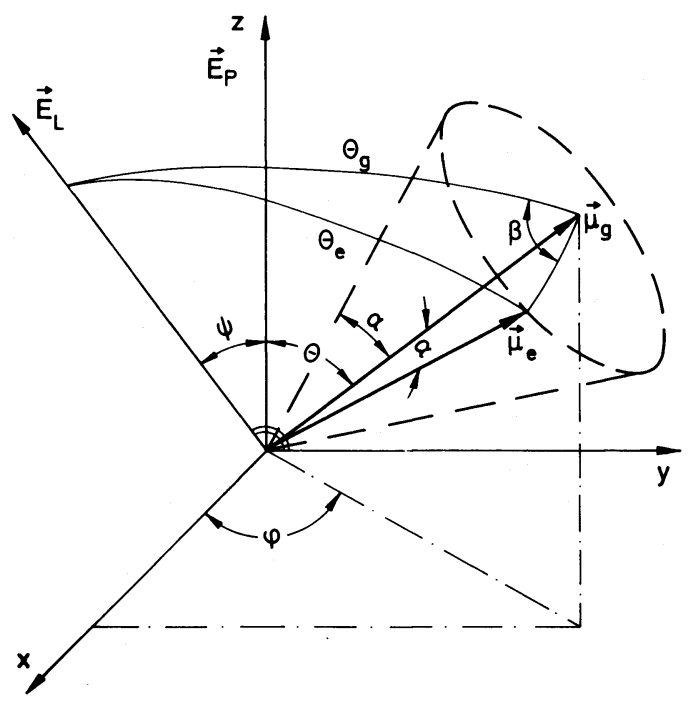

Fig. 2. Orientational arrangement of transition dipole moments $\boldsymbol{\mu}_{\mathrm{g}}, \boldsymbol{\mu}_{\mathrm{e}}$ and light pulses $\boldsymbol{E}_{\mathbf{P}}, \boldsymbol{E}_{\mathrm{L}}$. The $y$-axis is parallel to the pump $\left(\boldsymbol{E}_{\mathrm{P}}\right)$ and probe $\left(\boldsymbol{E}_{\mathrm{L}}\right)$ pulse propagation direction. 


$$
\begin{aligned}
\theta_{\mathrm{e}} & =\arcsin \left(\frac{\sin (\beta) \sin \left(\theta_{\mathrm{g}}\right)}{A}\right), \text { with } \\
A & =\sin \left\{\operatorname { a r c c o t } \left[\left(\sin (\alpha) \operatorname{cotan}\left(\theta_{\mathrm{g}}\right)\right.\right.\right. \\
& -\cos (\alpha) \cos (\beta)) / \sin (\beta)]\} .
\end{aligned}
$$

The angle $\theta_{\mathrm{g}}$ between $\boldsymbol{E}_{\mathrm{L}}$ and $\boldsymbol{\mu}_{\mathrm{g}}$ is obtained by plane trigonometric considerations:

$\theta_{\mathrm{g}}=\arccos [\sin (\psi) \sin (\theta) \cos (\varphi)+\cos (\psi) \cos (\theta)]$,

$\theta$ and $\varphi$ are the polar angles of $\boldsymbol{\mu}_{\mathrm{g}}$.

The transmission of a weak probe beam is found by integration over the sample length $l$ and over the molecular orientations $\left(\theta\right.$ and $\varphi$ for distribution of $\boldsymbol{\mu}_{\mathrm{g}}, \beta$ for the arrangement of $\boldsymbol{\mu}_{\mathrm{e}}$ with respect to $\boldsymbol{\mu}_{\mathrm{g}}$ ).

$$
\begin{gathered}
T_{\mathrm{e}}(\alpha, \psi)=\exp \left(-\frac{3 \sigma_{\mathrm{e}}}{\pi^{2}} \int_{0}^{l} \int_{0}^{\pi / 2} \sin (\theta) N_{3}(\theta, z)\right. \\
\left.\times \int_{0}^{\pi} \int_{0}^{\pi} \cos ^{2}\left(\theta_{\mathrm{e}}\right) \mathrm{d} \beta \mathrm{d} \varphi \mathrm{d} \theta \mathrm{d} z\right),
\end{gathered}
$$

$N_{3}(\theta, z)$ is the number density of molecules in level 3 . For the studies on rhodamine $6 \mathrm{G}, N_{3}(\theta, z)$ is calculated with the equation system of ref. [19] and the dye parameters of ref. [14]. (Eq. (5) of [19] was extended to include orientational anisotropy and reorientation [10]). The effects of amplified spontaneous emission are included. Calculations indicate that the amplified fluorescence becomes only slightly polarized under our experimental conditions (extension of eq. (11) of ref. [19]; amplified fluorescence duration $>$ reorientation time). The incluence of partial polarization of amplified emission on $N_{3}(\theta, \varphi, z)$ is negligibly small.

At $\psi=54.7^{\circ}\left(\tan ^{2}(\psi)=2\right)[11,20]$ one finds

$\frac{3}{\pi^{2}} \int_{0}^{\pi} \int_{0}^{\pi} \cos ^{2}\left[\theta_{\mathrm{e}}\left(\beta, \varphi, \theta, \alpha, \psi=54.7^{\circ}\right)\right] \mathrm{d} \beta \mathrm{d} \varphi=1$,

and eq. (4) reduces to

$$
\begin{aligned}
& T_{\mathrm{e}}\left(\alpha, \psi=54.7^{\circ}\right) \\
& \quad=\exp \left(-\sigma_{\mathrm{e}} \int_{0}^{l} \int_{0}^{\pi / 2} \sin (\theta) N_{3}(\theta, z) \mathrm{d} \theta \mathrm{d} z\right),
\end{aligned}
$$

which is independent of $\alpha$. Eq. (6) represents the transmission for an orientationally isotropic population with
$N_{3}(z)=\int_{0}^{\pi / 2} N_{3}(\theta, z) \sin (\theta) \mathrm{d} \theta$. This fact allows orientation free absorption measurements at $\psi=54.7^{\circ}$ [20].

The angle $\alpha$ between $\boldsymbol{\mu}_{\mathrm{g}}$ and $\boldsymbol{\mu}_{\mathrm{e}}$ is determined by comparing the measured transmissions $T_{\mathrm{e} \|}=T_{\mathrm{e}}\left(\psi=0^{0}\right)$ and $T_{\mathrm{e} \perp}=T_{\mathrm{e}}\left(\psi=90^{\circ}\right)$ with theory. It should be noted that the logarithmic transmission ratio $\ln \left(T_{\mathrm{e} \|}\right) / \ln \left(T_{\mathrm{e} \perp}\right)$ is independent of the excited state absorption crosssection $\sigma_{\mathrm{e}}$.

\section{Experiment}

The experimental setup is shown in fig. 3. Picosecond light pulses are generated in a modelocked Nd-phosphate glass laser [21]. Single light pulses are selected from the pulse train with an electrooptical switch and increased in energy with a Nd-glass amplifier. The light signals behind the amplifier have a duration of $\Delta t_{\mathrm{L}} \approx 6 \mathrm{ps}$ (FWHM), a bandwidth of $\Delta \nu_{\mathrm{L}} \approx 3 \mathrm{~cm}^{-1}$ (FWHM), and an energy of $\approx 5 \mathrm{~mJ}$. The second harmonic light generated in a KDP crystal (conversion efficiency $\approx 0.3$ ) is used as the pump pulse (frequency $\widetilde{\nu}_{\mathrm{P}}=18960 \mathrm{~cm}^{-1}$ ). The fundamental and second harmonic light pulses are separated with the harmonic beam splitter H1. Lens $\mathrm{L} 1(f=800 \mathrm{~mm})$ increases the peak intensity of the pump pulse (beam diameter at sample $\approx 4 \mathrm{~mm}$ ). The fundamental light pulse is reduced in energy with filter F1 and acts as probe pulse (frequency $\tilde{\nu}_{\mathrm{L}}=9480 \mathrm{~cm}^{-1}$ ). The time delay between probe and pump pulse is adjusted by the optical delay line DL. Lens L2 ( $f=600$ $\mathrm{mm}$ ) focuses the probe beam near to the sample. The probe beam diameter in the sample $(\approx 1 \mathrm{~mm})$ is small

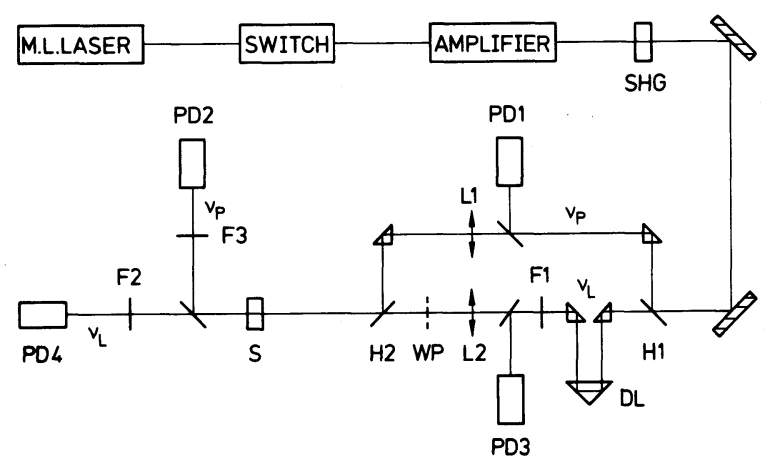

Fig. 3. Experimental set-up. SHG, KDP crystal for second harmonic generation. $\mathrm{H} 1, \mathrm{H} 2$, harmonic beam splitters. DL, optical delay line. F1-F3, filters. $L 1$, L2, lenses. WP, $\lambda / 2$ wave plate. S, dye sample. PD1-4, photodetectors. 
compared to the pump beam diameter. A constant excited state population is assumed across the probe beam area. The $\lambda / 2$ waveplate WP is put into the path for measurements with probe polarization parallel to pump pulse polarization. Both laser beams are reunited at the harmonic beam splitter H2. The peak intensity of the pump pulse is monitored by measuring its transmission through the sample with detectors PD1 and PD2 [19]. The probe pulse transmission is measured with photodetectors PD3 and PD4.

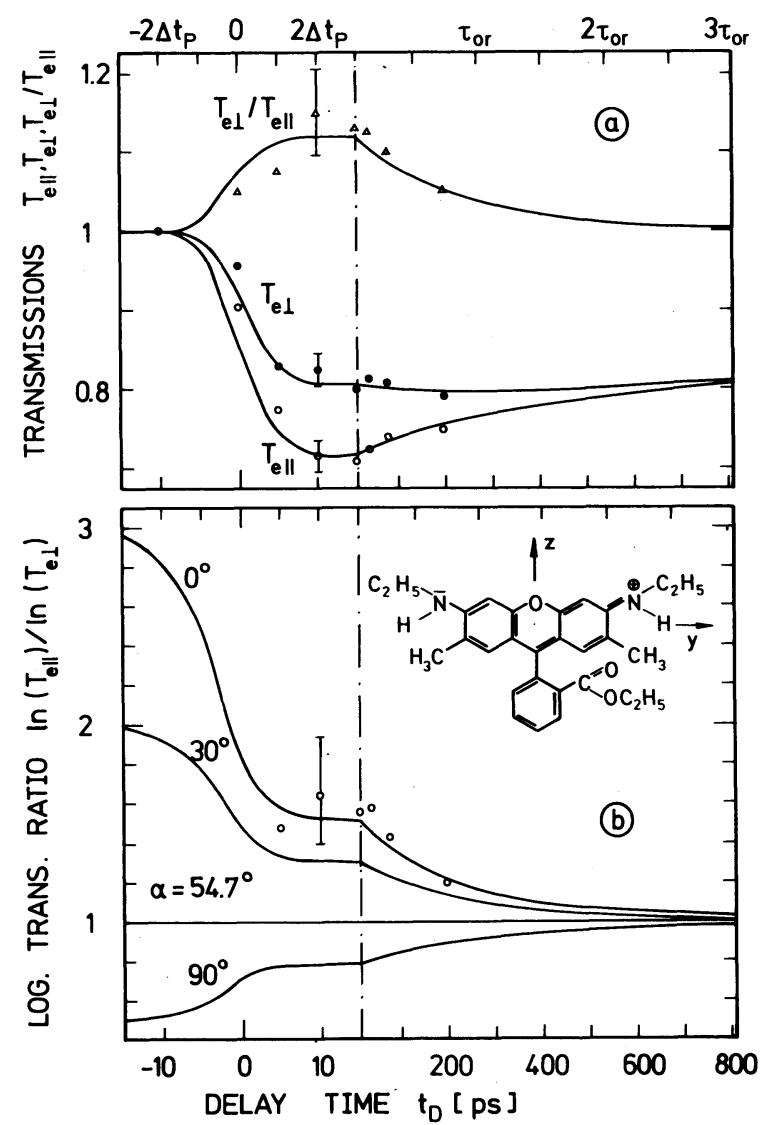

Fig. 4. (a) Probe pulse transmissions versus delay time. Curves are calculated for $\alpha=0$ with the experimental parameters $I_{0} \mathrm{P}=$ $10^{9} \mathrm{~W} / \mathrm{cm}^{2}, \Delta t_{\mathrm{P}}=5 \mathrm{ps}, \tau_{\mathrm{or}}=270 \mathrm{ps}, c=10^{-4} \mathrm{M}, l=5 \mathrm{~mm}$, $\sigma_{\mathrm{e}}=2 \times 10^{-17} \mathrm{~cm}^{2}$. Experimental points: open circles, $T_{\mathrm{e} \|}$; full circles, $T_{\mathrm{e} \perp}$; triangles, $T_{\mathrm{e} \perp} / T_{\mathrm{e} \|}$. (b) Logarithmic transmission ratio as a function of delay time. Curves are calculated for various angles $\alpha$, with the same parameters as in (a). Comparison with experimental points indicates $\alpha \approx 0^{\circ}\left(\lesssim 30^{\circ}\right)$. The structural formula of rhodamine $6 \mathrm{G}$ is inserted. Time scale is changed at $t_{\mathrm{D}}=3 \Delta t_{\mathrm{P}}=15$ ps. Error bars indicate standard deviation.

\section{Results}

The transmissions $T_{\mathrm{e} \|}$ and $T_{\mathrm{e} \perp}$ were measured for various delay times $t_{\mathrm{D}}$. For $t_{\mathrm{D}} \leqslant 20 \mathrm{ps}, 10^{-4}$ molar rhodamine $6 \mathrm{G}$ in ethanol was used in a sample of $5 \mathrm{~mm}$ length, while for $t_{\mathrm{D}}>20$ ps a $2 \mathrm{~cm}$ long cell with $2.5 \times 10^{-5}$ molar solution was applied to avoid enhanced depopulation of the $S_{1}$ state within the observation time due to stimulated emission initiated by feedback of fluorescence light from the cell windows. The pump pulse peak intensity was adjusted to about $I_{0 \mathrm{P}}=10^{9} \mathrm{~W} / \mathrm{cm}^{2}$ (see fig. 5). The effects of amplified spontaneous emission are included in the calculated curves of figs. 4 and 5, but they are negligibly small in our experiments $\left(t_{\mathrm{D}} \leqslant 200 \mathrm{ps}, N l=3 \times 10^{16} \mathrm{~cm}^{-2}\right)$ $[17,19]$. According to fig. 8 of ref. [19] the fluorescence lifetime is shortened from $4.2 \mathrm{~ns}$ to $2.5 \mathrm{~ns}$ by amplified spontaneous emission $\left(I_{0 \mathrm{P}}=10^{9} \mathrm{~W} / \mathrm{cm}^{2}\right)$.

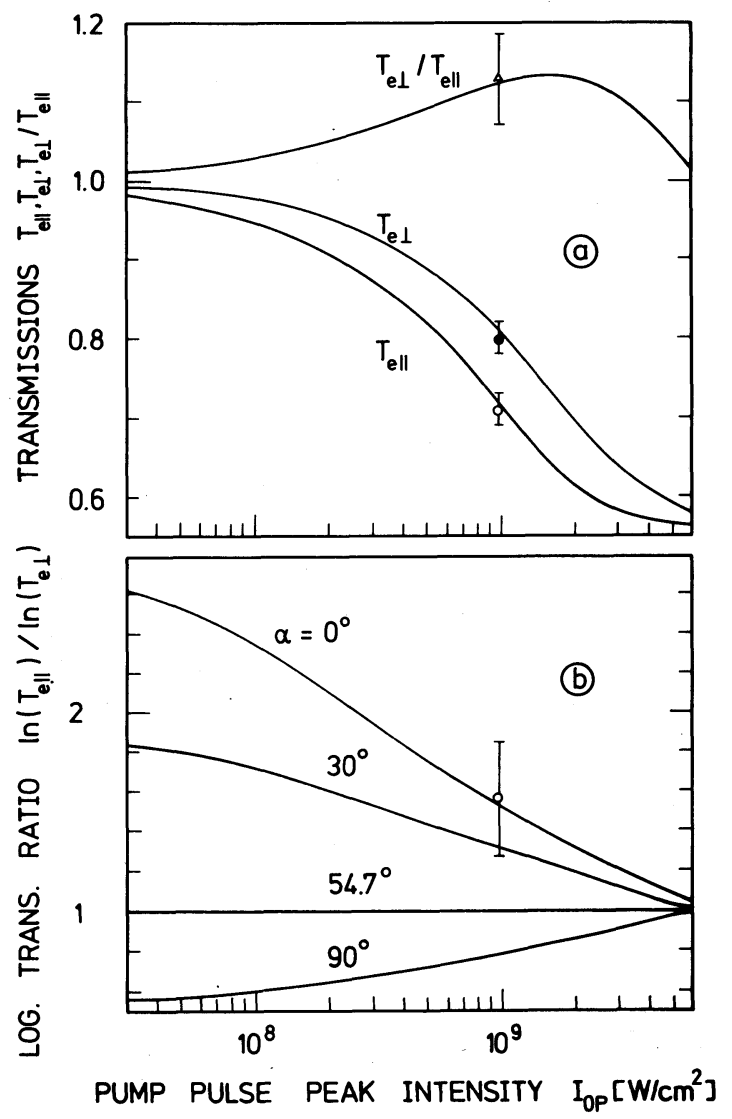

Fig. 5. Dependence of excited state transmissions on pump pulse peak intensity. Parameters as in fig. 4. 
Fig. 4 presents experimental points for $T_{\mathrm{e} \|}, T_{\mathrm{e} \perp}$, $T_{\mathrm{e} \perp} / T_{\mathrm{e} \|}$ and $\ln \left(T_{\mathrm{e} \|}\right) / \ln \left(T_{\mathrm{e} \perp}\right)$ at $I_{0 \mathrm{P}}=10^{9} \mathrm{~W} / \mathrm{cm}^{2}$ together with theoretical curves (eq. 4). The decrease of excited state transmissions $T_{\mathrm{e} \|}$ and $T_{\mathrm{e} \perp}$ around $t_{\mathrm{D}}$ $=0$ allows to determine zero delay between the pump and probe pulses and to measure pulse durations. The fast change of the $\ln \left(T_{\mathrm{e} \|}\right) / \ln \left(T_{\mathrm{e} \perp}\right)$ curves between $t_{\mathrm{D}}=-10 \mathrm{ps}$ and $+10 \mathrm{ps}$ is due to reducing the orientational anisotropy of level 3 with pump pulse excitation (bleaching effect). The slow decrease of $T_{\mathrm{e} \perp} / T_{\mathrm{e} \|}$ and $\ln \left(T_{\mathrm{e} \|}\right) / \ln \left(T_{\mathrm{e} \perp}\right)$ after excitation $\left(t_{\mathrm{D}} \geqslant 10 \mathrm{ps}\right)$ is determined by orientational redistribution $\left(\tau_{\text {or }} \approx\right.$ $270 \mathrm{ps})$.

Fig. 5 shows calculated curves of the excited state transmissions $T_{\mathrm{e} \|}, T_{\mathrm{e} \perp}$ and the ratios $T_{\mathrm{e} \perp} / T_{\mathrm{e} \|}$ and $\ln \left(T_{\mathrm{e} \|}\right) / \ln \left(T_{\mathrm{e} \perp}\right)$ versus pump pulse peak intensity $I_{0 \mathrm{P}}$ for a delay time of $t_{\mathrm{D}}=15 \mathrm{ps}$. The transmissions start at $T_{\mathrm{e} \|}=T_{\mathrm{e} \perp}=1$ at low intensities and decrease to a limiting value $T_{\mathrm{e} \|}=T_{\mathrm{e} \perp} \approx \exp \left[-\sigma_{\mathrm{e}} N l\right] \approx 0.55$ at high intensities were the ground state is completely bleached $\left(\sigma_{\mathrm{e}}=2 \times 10^{-17} \mathrm{~cm}^{2}[12], N=6 \times 10^{16} \mathrm{~cm}^{-3}, l=0.5\right.$ $\mathrm{cm})$. The transmission ratio $T_{\mathrm{e} \perp} / T_{\mathrm{e} \|}$ has a maximum around $I_{0 \mathrm{P}}=2 \times 10^{9} \mathrm{~W} / \mathrm{cm}^{2}$ (largest difference between the measured quantities $T_{\mathrm{e} \perp}$ and $\left.T_{\mathrm{e} \|}\right)$. In this intensity region the highest accuracy is obtained in the measurement of the orientation angle $\alpha$. The logarithmic transmission ratio (independent of $\sigma_{\mathrm{e}}$ ) spans a range from 3 $(\alpha=0)$ to $0.5\left(\alpha=90^{\circ}\right)$ at low intensities $\left(N_{3}(\theta) \propto\right.$ $\cos ^{2}(\theta)$ ) and shrinks to a point at high intensities (isotropic distributions of $N_{3}(\theta)$ when all molecules are excited to level 3).

A comparison of the experimental points with the calculated curves indicates a parallel orientation of $\boldsymbol{\mu}_{\mathrm{g}}$ and $\boldsymbol{\mu}_{\mathrm{e}}\left(\alpha \lesssim 30^{\circ}\right.$ may be resolved). It should be noted that the orientation angle was measured for molecules with a small excited state absorption cross-section of $\sigma_{\mathrm{e}}=2 \times 10^{-17} \mathrm{~cm}^{2}$. The method is more accurate for molecules with hígher excited state absorption crosssections.

\section{Discussion}

The orientation of the transition dipole moments is determined by the wavefunctions of the involved states and the molecular structure. The symmetry of rhodamine $6 \mathrm{G}$ is $C_{2 \mathrm{~V}}$.
In fig. $1 \mathrm{~b}$ calculated transition dipole moments $\left|\mu_{y}\right|$ (along longitudinal axis linking the amino-chromophors, see inset in fig. $4 \mathrm{~b}$ ) and $\left|\mu_{z}\right|$ for $\mathrm{S}_{0}-\mathrm{S}_{i}$ transitions are plotted at the energy positions of $S_{i}(i=1$ to 7$)$. The values of the transition moments were determined by Praiser-Pass-Pople (PPP-) calculations [12]. The directions of the calculated $\mathrm{S}_{0}-\mathrm{S}_{i}$ transition moments lead to the symmetry representation of the energy levels given in fig. 1a. This symmetry assignment allows to determine the orientation of excited state transition dipole moments. For the investigated $\mathrm{S}_{1}-\mathrm{S}_{4}$ transition one finds $\mu\left(\mathrm{S}_{1}-\mathrm{S}_{4}\right)\left\|\boldsymbol{\mu}\left(\mathrm{S}_{0}-\mathrm{S}_{1}\right)\right\| y$-axis in agreement with our experiments (in both cases excited between $A_{1}$ and $B_{2}$ states).

The conventional $S_{1} \rightarrow S_{0}$ fluorescence polarization spectrum of Jacobi and Kuhn [22] for rhodamine B is redrawn in fig. 1b. (The fluorescence spectrum of rhodamine $6 \mathrm{G}$ is stated to be similar [7]). The orientations of the transition dipole moments obtained from the polarization spectrum agree with the calculated directions and lead to the same symmetry assignments as the PPP-calculations. Especially, it is found that $\boldsymbol{\mu}\left(\mathrm{S}_{0}-\mathrm{S}_{4}\right)$ is nearly perpendicular to $\boldsymbol{\mu}\left(\mathrm{S}_{0}-\mathrm{S}_{1}\right)$. The reduced polarization anisotropy $(P \approx-0.2$ instead of -0.33 for perpendicular orientation) is assumed to be due to deformational and torsional vibrations which are involved in the excitation, relaxation and emission processes $[25,1,10]$.

Additional support of the symmetry assignment of the $S_{1}$ and $S_{4}$ levels is given by two-photon absorption spectroscopy $[23,24]$ where the strong $S_{0}-S_{4}$ twophoton absorption cross-section suggests a transition between two $A_{1}$ states [23]. The two-photon absorption spectroscopy with a set of differently polarized pump and probe beams allows an excited state symmetry assignment of fluids [27,28]. This technique was applied to determine the symmetry representation of excited states of 1-chloronaphthalene and benzene $[27,29]$. To our knowledge this technique has not been applied to rhodamine dye solutions up to now.

The $S_{i>1} \rightarrow S_{0}$ fluorescence polarization spectra of rhodamine $6 \mathrm{G}$ after two-step absorption to $\mathrm{S}_{4}$ [5-8] were found to be similar to the conventional excitation fluorescence polarization spectra. These spectra can be understood by assuming $\boldsymbol{\mu}\left(\mathrm{S}_{1}-\mathrm{S}_{4}\right) \| \boldsymbol{\mu}\left(\mathrm{S}_{0}-\mathrm{S}_{1}\right)$ and $\boldsymbol{\mu}\left(\mathrm{S}_{0}-\mathrm{S}_{4}\right) \perp \boldsymbol{\mu}\left(\mathrm{S}_{0}-\mathrm{S}_{1}\right)$.

In refs. [6] and [7] the orientation of the $S_{1}-S_{4}$ transition moment was reported to be parallel to the 
$\mathrm{S}_{0}-\mathrm{S}_{1}$ transition moment by analysing fluorescence yield measurements. Rhodamine $6 \mathrm{G}$ molecules were transversely excited in a laser resonator with $530 \mathrm{~nm}$ and $1.06 \mu \mathrm{m}$ laser pulses. The stated orientation of $\boldsymbol{\mu}\left(\mathrm{S}_{1}-\mathrm{S}_{4}\right)$ is in agreement with our measurements.

\section{Conclusions}

The orientation of $\boldsymbol{\mu}\left(\mathrm{S}_{1}-\mathrm{S}_{4}\right)$ was found to be parallel to $\mu\left(S_{0}-S_{1}\right)$ and perpendicular to $\mu\left(S_{0}-S_{4}\right)$ in rhodamine 6G. The described absorption spectroscopic technique with picosecond light pulses may be extended to measure polarization spectra over a wide frequency range by using picosecond light continua as probe beams [26].

\section{Acknowledgement}

The authors thank Professor M. Maier for valuable discussions. They gratefully acknowledge financial support by the Deutsche Forschungsgemeinschaft.

\section{References}

[1] P.P. Feofilov, The physical basis of polarized emission (Consultants Bureau, New York, 1961).

[2] F. Dörr, Angew. Chem. 78 (1966) 457.

[3] G. Weber, in: Fluorescence and phosphorescence analysis, ed. D.M. Hercules (Interscience, New York, 1966) p. 217.

[4] A.C. Albrecht, J. Mol. Spectry. 6 (1961) 84.

[5] H.-B. Lin and M.R. Topp, Chem. Phys. Letters 47 (1977) 442.

[6] A.V. Aristov and V.S. Shevandin, Opt. Spectrosc. 44 (1978) 276.
[7] A.V. Aristov and V.S. Shevandin, Opt. Spectrosc. 45 (1978) 825.

[8] V.L. Bogdanov, V.P. Klochkov, and B.S. Neporent, Opt. Spectrosc. 43 (1977) 701.

[9] D. Madge, J. Chem. Phys. 68 (1978) 3717.

[10] A. Penzkofer and W. Falkenstein, Chem. Phys. Letters 44 (1976) 547.

[11] H.E. Lessing and A. von Jena, Chem. Phys. Letters 59 (1978) 249.

[12] J. Wiedmann and A. Penskofer, submitted to Nuovo Cimento.

[13] T.J. Chuang and K.B. Eisenthal, Chem. Phys. Letters 11 (1971) 368.

[14] W. Falkenstein, A. Penzkofer and W. Kaiser, Optics Comm. 27 (1978) 151.

[15] A. Kawski, Z. Naturforschg. 18a (1963) 961.

[16] D.N. Dempster, J. Photochem. 2 (1973) 343.

[17] A. Penzkofer, W. Falkenstein, and W. Kaiser, Chem. Phys. Letters 44 (1976) 82.

[18] I.N. Bronstein and K.A. Semendjajew, Taschenbuch der Mathematik (Deutsch, Zürich, 1967) p. 164.

[19] A. Penzkofer and W. Falkenstein, Opt. Quant. Electr. 10 (1978) 399.

[20] H.E. Lessing and A. von Jena, Chem. Phys. Letters 42 (1976) 213.

[21] A. Laubereau and W. Kaiser, Opto-Electronics 6 (1974) 1.

[22] H. Jacobi and H. Kuhn, Z. Elektrochem. 66 (1962) 46.

[23] J.P. Hermann and J. Ducuing, Optics Comm. 6 (1972) 101.

[24] E.B. Aslanidi and E.A. Tikhonov, Opt. Spectrosc. 37 (1974) 446.

[25] A. Jablonski, Acta Phys. Polon. 10 (1950) 3.

[26] A. Penzkover and W.Ka iser, Opt. Quant. Electr. 9 (1977) 315.

[27] P.R. Monson and W.M. McClain, J. Chem. Phys. 53 (1970) 29.

[28] W.M. McClain, J. Chem. Phys. 55 (1971) 2789.

[29] P.R. Monson and W.M. McClain, J. Chem. Phys. 56 (9172) 3817 . 